This item was submitted to Loughborough's Research Repository by the author.

Items in Figshare are protected by copyright, with all rights reserved, unless otherwise indicated.

\title{
On the dynamic response of an instrumented headform for alternative mounting stiffnesses when subjected to ballistic impacts
}

\section{PLEASE CITE THE PUBLISHED VERSION}

https://doi.org/10.1177/1754337117703574

\section{PUBLISHER}

Sage (@ The Authors)

\section{VERSION}

AM (Accepted Manuscript)

\section{PUBLISHER STATEMENT}

This work is made available according to the conditions of the Creative Commons Attribution-NonCommercialNoDerivatives 4.0 International (CC BY-NC-ND 4.0) licence. Full details of this licence are available at: https://creativecommons.org/licenses/by-nc-nd/4.0/

\section{LICENCE}

CC BY-NC-ND 4.0

\section{REPOSITORY RECORD}

Stone, Ben W., Andy Harland, James P. Jones, Sean Mitchell, Paul Sherratt, Craig A. Ranson, and Ben Halkon. 2017. "On the Dynamic Response of an Instrumented Headform for Alternative Mounting Stiffnesses When Subjected to Ballistic Impacts". Loughborough University. https://hdl.handle.net/2134/23170. 


\section{Article type}

2 Original Article

\section{Corresponding author info}

4 Corresponding Author:

5 Dr Benjamin J. Halkon, Wolfson School. Mechanical, Electrical and Manufacturing Engineering,

6 Loughborough University, Ashby Road, Loughborough, Leics., LE11 3TU

7 Email: b.j.halkon@lboro.ac.uk

8 Article title

9 On the dynamic response of an instrumented headform for alternative mounting

10 stiffnesses when subjected to ballistic impacts

\section{Authors}

12 Ben W. Stone ${ }^{1}$, Andy R. Harland ${ }^{1}$, James A. Jones ${ }^{1}$, Séan R. Mitchell ${ }^{1}$, Paul J. Sherratt ${ }^{1}$, Craig A. Ranson ${ }^{2}$

13 and

14 Ben J. Halkon ${ }^{1}$

15 'Wolfson School. Mechanical, Electrical and Manufacturing Engineering, Loughborough University,

16 Loughborough, UK.

$17{ }^{2}$ Cardiff School of Sport, Cardiff Metropolitan University-UWIC, Cardiff, UK and English institute of Sport,

18 Alan Turing Way, Manchester, Uk

\section{Abstract}

\section{Abstract}

The current British Standard for head protectors for cricketers has been recently revised to include a projectile-based battery of tests, the intention being to ensure that a certified helmet will also prevent contact of the ball or grille with the specified headform facial region. The purpose of this study was to characterise the dynamic response of the headform to direct ballistic impacts for alternative headform mounting arrangements. On the one hand, and in accordance with the relevant sections of the Standard, what might be described as a "Constrained" set-up was evaluated while, on the other, an arrangement with significantly reduced stiffness, in line with that previously reported for the passive human neck, was subject to equivalent appraisal.

28

For each mounting scenario, an air cannon was used to project a cricket training ball at three speeds toward the instrumented headform at three locations with five repeats per speed/location combination. Highrate/resolution video and piezo-electric accelerometer data were collected and processed to determine the headform response. While differences between specific ball impact speed and location scenarios are set out in detail later in the article, overall observations are summarised as follows. From a ball-headform contact duration standpoint, video derived results showed ranges of $1.30-1.45 \mathrm{~ms}$ (Constrained) vs. 1.26 - 1.41 ms. Maximum ball deformations the timing of which enabling the event to be subdivided into "loading" and "unloading" phases, were found to be $82.5-86.2 \%$ (Constrained) vs. $82.8-86.4 \%$ of original ball diameter, mean peak headform accelerations during loading were found to be $860-1615 \mathrm{~m} / \mathrm{s}^{2}$ (Constrained) vs. 967 $-1638 \mathrm{~m} / \mathrm{s}^{2}$ while headform speeds at the end of the loading phase were found to be $0.5-0.92 \mathrm{~m} / \mathrm{s}$ (Constrained) vs. $0.54-0.93 \mathrm{~m} / \mathrm{s}$. Differences between headform response for the two mounting arrangements were observed to be more substantial during the loading rather than unloading phase. 


\title{
1 On the dynamic response of an instrumented headform 2 for alternative mounting stiffnesses when subjected to 3 ballistic impacts
}

\author{
4 Ben Stone, Andy Harland, James Jones, Séan Mitchell, \\ 5 Paul Sherratt, Craig Ranson and Ben Halkon \\ 6 Abstract
}

7 The current British Standard for head protectors for cricketers has been recently revised

8 to include a projectile-based battery of tests, the intention being to ensure that a certified

9 helmet will also prevent contact of the ball or grille with the specified headform facial

10 region. The purpose of this study was to characterise the dynamic response of the

11 headform to direct ballistic impacts for alternative headform mounting arrangements. On

12 the one hand, and in accordance with the relevant sections of the Standard, what might be

13 described as a "constrained" set-up was evaluated while, on the other, an arrangement

14 with significantly reduced stiffness, in line with that previously reported for the passive

15 human neck, was subject to equivalent appraisal.

16 For each mounting scenario, an air cannon was used to project a cricket training ball at

17 three speeds toward the instrumented headform at three locations with five repeats per

18 speed/location combination. High-rate/-resolution video and piezoelectric accelerometer

19 data were collected and processed to determine the headform response. While differences

20 between specific ball impact speed and location scenarios are set out in detail later in the

21 article, overall observations are summarised as follows. From a ball-headform contact

22 duration standpoint, video derived results showed ranges of $1.30-1.45 \mathrm{~ms}$ (Constrained)

23 vs. $1.26-1.41 \mathrm{~ms}$. Maximum ball deformations, the timing of which enabling the event

24 to be subdivided into "loading" and "unloading" phases, were found to be $82.5-86.2 \%$

25 (constrained) vs. $82.8-86.4 \%$ of original ball diameter, mean peak headform

26 accelerations during loading were found to be $860-1615 \mathrm{~m} / \mathrm{s}^{2}$ (Constrained) vs. 967 -

$271638 \mathrm{~m} / \mathrm{s}^{2}$ while headform speeds at the end of the loading phase were found to be 0.5 -

$280.92 \mathrm{~m} / \mathrm{s}$ (Constrained) vs. $0.54-0.93 \mathrm{~m} / \mathrm{s}$. Differences between headform response for

29 the two mounting arrangements were observed to be more substantial during the loading

30 rather than unloading phase. 


\section{Introduction}

2 Head protection is commonly available in many sports. A participant's use of a helmet

3 may be mandated in the rules of a competition, for example in Formula $1^{\mathrm{TM}}$ motor racing ${ }^{1}$

4 and snowboarding ${ }^{2}$. In some sports, however, helmet use may be permitted in the rules,

5 but ultimately will be a personal decision aimed at reducing the risk of incurring injury.

6 Until recently this latter scenario was the case in cricket ${ }^{3}$. However, largely in response to

7 recent changes to the nature of the game ${ }^{4}$ and most certainly one particularly tragic

8 event $^{5}$, the England and Wales Cricket Board has made the use of a helmet mandatory for

9 batsmen from the 2016 season and onwards ${ }^{6}$. In extreme cases, the function of a helmet is

10 to prevent death or life-changing injury, although, in many cases, the role of a helmet is

11 to protect against more minor injury, disfigurement or discomfort.

12 Walker et al. ${ }^{7}$ reported that, over a five-year period in New Zealand, $21 \%$ of all injuries

13 resulting in hospitalisation for professional and recreational cricketers were sustained to

14 the head. In elite-level cricket, the speed of the ball can exceed $90 \mathrm{mph}$ when bowled or

15 thrown ${ }^{4}$, or, of course, higher still when hit by the bat. While lower speeds are inevitably

16 more normal at recreational levels of the game, direct contact between the ball and the

17 head or contact as a result of helmet deformation can still cause injuries, such as

18 concussions, eye injuries, facial fractures, lacerations ${ }^{8}$ or even death ${ }^{9,10}$. Safety is not, of

19 course, the only consideration when developing (sports) helmets; comfort and the linked

20 ability of the wearer to effectively perform are also highly important factors ${ }^{11}$ with

21 designers and engineers typically having to compromise. In cricket specifically, a strong

22 tradition further results in the styling and aesthetics also being of importance. Indeed, a

23 helmet providing complete, all-round protection for a cricket batsman is unlikely to be 
1 utilised should it restrict vision, prohibit optimal performance or drastically differ from

2 the traditional appearance of cricket headwear.

3 To be sold legally (in Europe), protective helmets must satisfy current standards, and the

4 tests therein, and thereby adhere to certain quality controls ${ }^{12}$. In the specific context of

5 cricket helmets (for batsmen), the scope for the relevant Standard, BS 7928: 2013 ${ }^{13}$,

6 includes specification of "the requirements for the materials, construction, markings and

7 information to be supplied for head protectors". Additionally, and with appropriate

8 reference to other generic protective helmets performance test $\operatorname{standards}^{14}$, BS 7928: 2013

9 "specifies the methods to assess the impact attenuation properties during a drop test of

10 the helmet and the protection provided against a ball or faceguard contacting a specified

11 no contact zone of the face during a projectile test”. Such tests must represent an

12 appropriate performance benchmark at reasonable cost to avoid excessive development

13 costs being passed on to the consumer. For these reasons, relatively simple mechanical

14 arrangements, often based on energy equivalent collisions between objects of increased

15 mass at reduced velocities (such as the free-fall test described by Johnson ${ }^{15}$ ) are typically

16 favoured. This is largely due to the relative ease with which i) controlled, repeatable tests

17 can be conceived and executed, ii) the necessary observations can be made and iii) the

18 pass/fail criteria can be defined; product performance can thereby be confidently

19 evaluated.

20 The accepted impact attenuation performance battery of tests within BS 7928: 2013

21 follows such a path. Here a falling (magnesium alloy ${ }^{16}$ ) headform method ${ }^{14}$ is proposed

22 in which " $A$ steel anvil, with a hemispherical striking face with a diameter of (73 \pm 1$)$

$23 \mathrm{~mm} " 13$ is specified. The required impact speed (for a size 575 headform) must be 2.53- 
$12.63 \mathrm{~m} / \mathrm{s}$ and the resultant deceleration, "measured by a tri-axial accelerometer located

2 within the headform" of the helmeted headform, must be no more than $250 \mathrm{~g}$. Four

3 impact sites per helmet type must be tested with a single impact conducted per helmet

4 sample at each impact site.

5 Such simplified equivalents have, however, been shown to be less valid for the case of a

6 ball projected at the gap between the peak (the protrusion just above the eye-line of the

7 shell of the helmet) and the grille (face protector commonly made of metal) of a typical

8 cricket helmet ${ }^{17}$. For this reason, it was a projectile, rather than a falling mass or

9 headform test, that was ultimately incorporated into BS 7928: 2013; revised in response

10 to recent high-profile injuries ${ }^{4}$. While maintaining the impact attenuation test element, the

11 intention of the inclusion of the facial contact projectile test is to ensure that helmets are

12 also capable of preventing facial contact, either directly from ball penetration of the peak-

13 grille gap or as a result of deformation of the faceguard following ball impact. To enable

14 this facial-contact projectile test, a "ball, that has experienced no more than 20 impacts...

15 [with] a diameter of between $71 \mathrm{~mm}$ and $73 \mathrm{~mm} .$. [and] mass... between $140 \mathrm{~g}$ and 150

$16 \mathrm{~g}$ for adult helmets... [is projected] at velocities up to $(28 \pm 3) \mathrm{m} / \mathrm{s} .$. [ [to impact] no

17 greater than $10 \mathrm{~mm}$ from the expected target location" ${ }^{\prime 13}$. Five target locations are

18 specified, three along the helmet centreline with two laterally. Determination of whether

19 or not the ball or the grille has come into contact with the headform is determined by the

20 use of contact indicator, e.g. "Developer" spray commonly used in crack/flaw detection.

21 Like the impact attenuation drop test, the facial contact projectile test also requires that

22 the helmet be fitted to a headform albeit, in this case, without the inclusion of the

23 accelerometer. Clearly the headform must be mounted by some means and there may be 
1 debate over the exact manner in which to do this so as to optimise "biofidelity", including

2 whether or not a representation of the neck should be incorporated; the means by which

3 this fitting should be achieved is not explicit in BS 7928: 2013. The purpose of this study

4 was, therefore, to evaluate the behaviour of two near-extreme examples of headform

5 mounting arrangement in terms of their influence on headform dynamics during and after

6 an un-helmeted ballistic impact.

7 The relationship between headform dynamics (and the measures by which they can be

8 defined for such an impact scenario) and the performance of a helmeted headform in

9 terms of preventing facial contact may be questioned. The intention of this study was,

10 however, more fundamental in terms of aiming to establish whether differences exist

11 between these two near-extreme arrangements during and shortly after ballistic impact

12 for the sake of better informing future research and development as well as standard tests

13 including both facial contact and impact attenuation projectile tests. The research

14 described herein uses state-of-the-art processes that are arguably not at the disposal of nor

15 practically applicable for typical test houses that determine whether or not cricket helmets

16 are safe. This intention is not, however, to have future Standards adjusted to require the

17 use of such processes but more to complement existing capabilities and glean additional

18 insights in support of the good work already being done. This paper will, for the first

19 time, report differences between ball/headform impact dynamics that result from

20 alternative headform mounting and ball impact speed/location during sports relevant

21 impacts, specifically those typical to cricket. 


\section{Methodology}

\section{Experimental arrangement and data collection}

4 The bespoke experimental arrangement employed in this study allowed for the 5 suspension mounting of a BS EN 960: $2006^{16}$ instrumented magnesium alloy headform, 6 size 575 (mass $4.7 \mathrm{~kg}$ ) in two alternative scenarios, as shown in Figure 1. In the,

7 "Constrained" (deliberately not rigid because some compliance is inevitable and, indeed, 8 desirable to limit the high shock when the impact occurs) scenario shown in Figure 1a,

9 the headform was post-mounted via a clamp arrangement incorporating a stiff anti-

10 vibration type bushing onto a grounded extruded aluminium frame. The bushing, shown

11 in detail in Figure 1c, includes a silicon rubber element which enables constraint of the

12 headform, while allowing some rotation about a pivot, the axis of which is $105 \mathrm{~mm}$ from

13 the base of the headform.

14 In the alternative, "Unconstrained" scenario, the headform was inverted and suspended 15 with bungee cords (10-mm diameter, $35-\mathrm{N}$ pre-test tension) attached to the base as per

16 Figure 1b. Attachment and alignment of the bungees require compromise with the ideal

17 scenario being one in which the suspension is aligned normal to the direction of travel 18 such that, upon disturbance from rest, there is no immediate bungee extension and, 19 therefore, no immediate restoring force. This compromise comes, however, at the 20 expense of readily repeatable positioning of the headform prior to each impact event and 21 some deviation from this ideal is therefore required ${ }^{18}$.

22 The absolute stiffness values of the two suspension scenarios were experimentally 23 determined by applying a series of torques to the headform while measuring the resulting 24 angular displacement. Practically this determination of suspension stiffness was achieved 
1 by applying a measured force, initially along the z-axis, at the reference plane (see Figure

2 2a) while measuring the resulting angular displacement ( $\alpha$ in Figure 2a) using image

3 processing. As angular displacement occurred, the line of action of the applied force was

4 adjusted so that it maintained a direction that was in-line with and directly along the

5 reference plane. For the Constrained scenario the stiffness was determined to be $5.6 \mathrm{~N}$ -

$6 \mathrm{~m} / \mathrm{deg}$, while, for the Unconstrained, a corresponding value of $0.2 \mathrm{~N}-\mathrm{m} / \mathrm{deg}$ was

7 established. This latter value is within the range of the passive stiffness of the human

8 neck, $0.03-0.3 \mathrm{~N}-\mathrm{m} / \mathrm{deg}^{19}$.

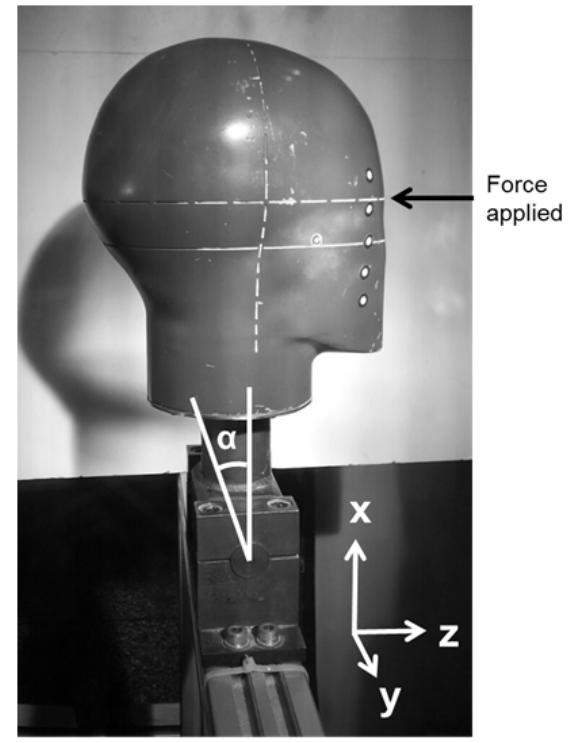

(a)

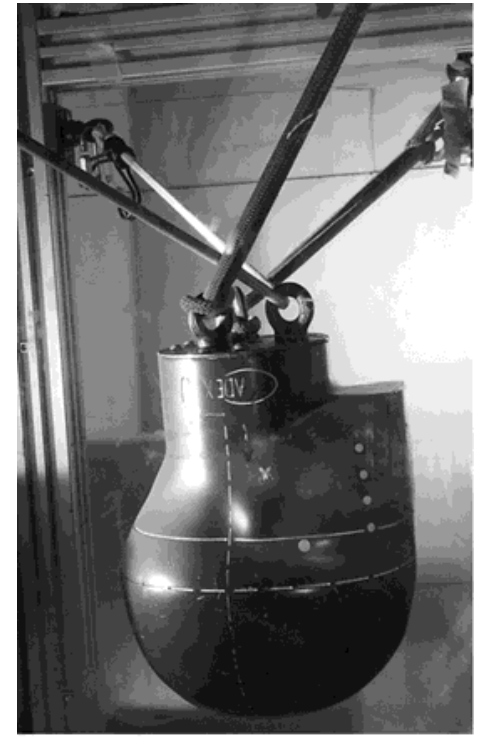

(b)
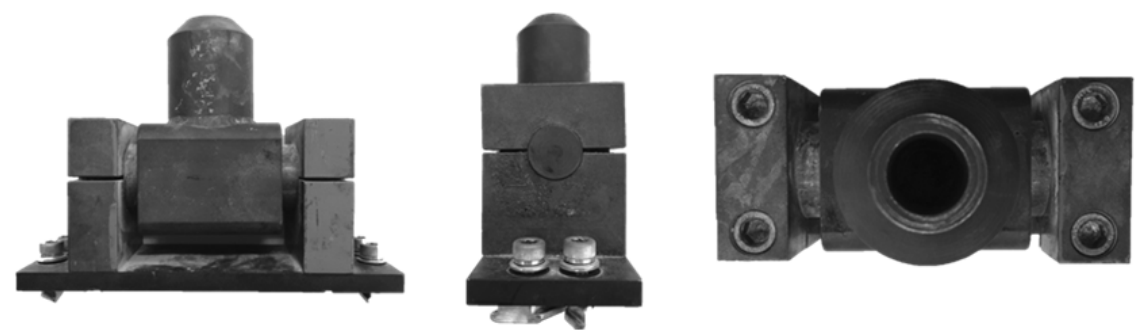

(c)

Figure 1. Alternative headform mounting arrangements; (a) "Constrained" via bushed mounting to a grounded frame, (b) "Unconstrained" with bungee cords between base and frame and (c) detailed images of the Constrained arrangement mounting. 


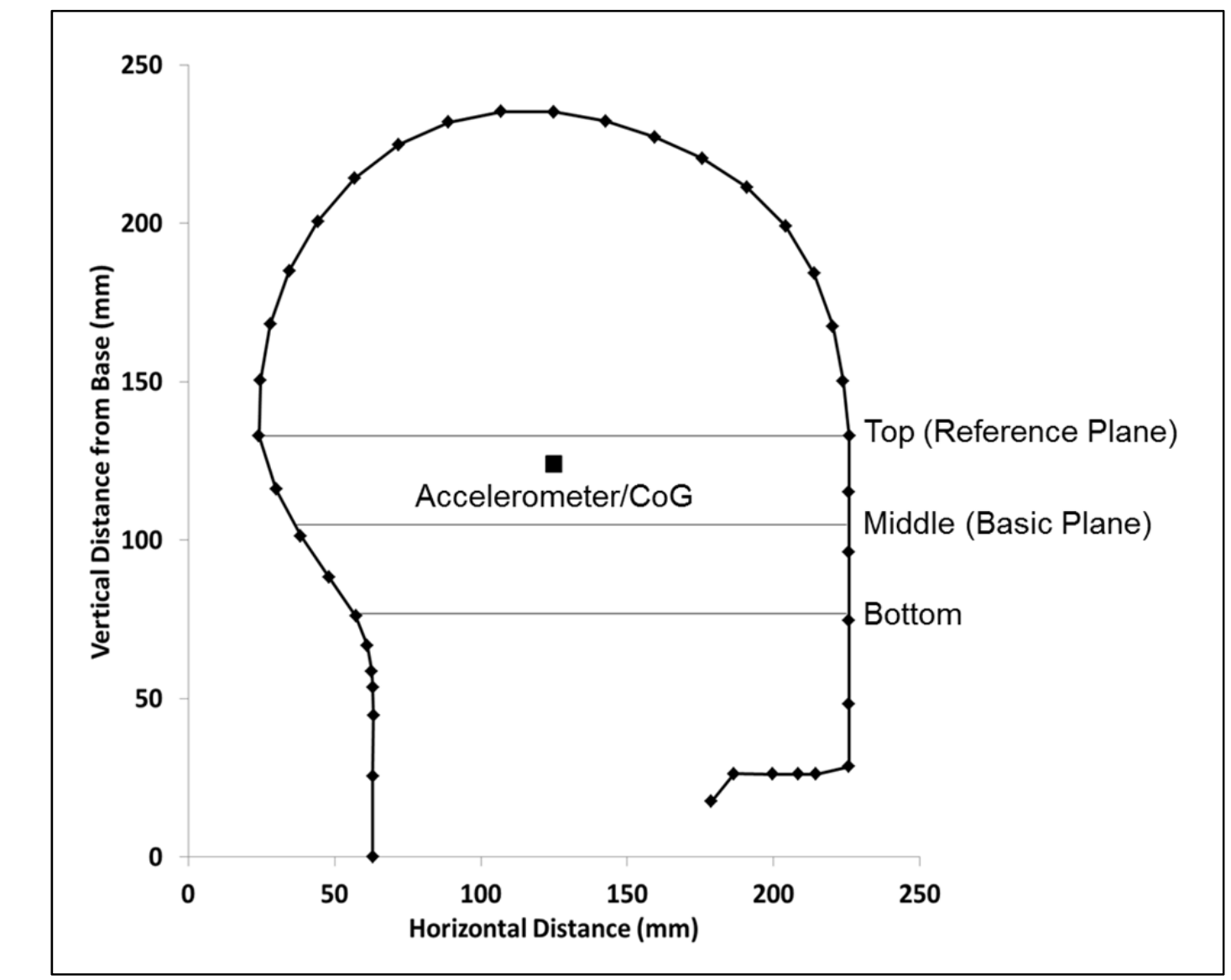

2 Figure 2. Section view of headform through the sagittal plane with "Top", "Middle" and

3 "Bottom" impact locations indicated as well as the accelerometer location.

4 Throughout all tests the same type of "BOLA"TM cricket training ball was used (mass

5 nominally $150 \mathrm{~g}$, diameter nominally $71 \mathrm{~mm}$ ), as specified in BS 7928: $2013^{13}$ for the

6 Facial contact projectile test. A pressurised air cannon was used to project the ball toward

7 the headform impact location at three speeds, nominally 28,25 and $22 \mathrm{~m} / \mathrm{s}$, the first two

8 also in line with those specified in the Standard. The experimental arrangement allowed

9 for the vertical repositioning of the headform relative to the cannon such that impacts

10 could be arranged to occur at three locations, nominally $136 \mathrm{~mm}$ ("Top"), $108 \mathrm{~mm}$

11 ("Middle") and $80 \mathrm{~mm}$ ("Bottom") from the base of the headform as shown in Figure 2.

12 The Top and Middle impact locations are aligned with the headform "Reference" and 
1 "Basic" planes while the Bottom is the same distance from the Middle as the Top is but

2 in the opposite direction. While the Top and the Middle impact locations and 28- and 25-

$3 \mathrm{~m} / \mathrm{s}$ impact speeds are in line with the specified Standard, the Bottom and 22-m/s impact

4 condition were included with a view to increasing any possibility to interpolate or indeed

5 extrapolate from the results determined to other speeds and/or impact locations in the

6 future.

7 It is worth noting at this point that, in the Standard, the helmeted headform positioning is

8 specified such that the ball ideally makes contact with the peak and grille simultaneously,

9 thereby maximising the chance of penetration occurring. Taking into account the variable

10 design of commercially available helmets, this "simultaneous impact" scenario

11 practically amounts to an impact location somewhere between the Reference and Basic

12 planes, hence the choice of these two target impact locations. It is also worth noting that a

13 helmeted headform, being more deformable upon ball impact, will result in an extended

14 impact duration compared to an un-helmeted headform. This extended duration impact

15 will include associated alternative characteristics including impulse and energy transfer

16 although these may or may not lead to significant differences in equivalent performance

17 to the scenarios investigated in this study.

18 A PCB 356B21 tri-axial piezoelectric accelerometer was rigidly fitted inside the

19 headform using the headform manufacturer supplied stud mounting arrangement. The

20 accelerometer was located at the headform centre of gravity (i.e. on the central axis,

$21 \quad 127 \mathrm{~mm}$ from the base) and orientated relative to the headform such that the three

22 acceleration measurement components were aligned with the directions shown in

23 Figure 1a. After the signal was conditioned using a DJB Instruments CV9 IEPE 
1 amplifier, the accelerometer voltage signals were captured using a LeCroy WaveJet 324

2 digital oscilloscope with a sample frequency of $50 \mathrm{MHz}$ specified as such so as to yield

3 substantial temporal resolution (with subsequent down-sampling if necessary readily

4 possible) for the anticipated circa 1-2 ms duration impact events. Accelerometer

5 sensitivities were determined in advance using a B\&K Type 4294 accelerometer

6 calibrator with the $x$-, $y$ - and $z$-directions being $1.18,1.22$ and $1.17 \mathrm{mV} / \mathrm{ms}^{-2}$, respectively.

7 Three Arri Pocket Par 400 lights were used to illuminate the testing area while a Photron

8 FastCam SA1 mono high-frame-rate camera, operating at $50 \mathrm{kHz}(448 \times 224$ pixels

9 spatial resolution), was positioned lateral and perpendicular to the plane of movement, as

10 can be seen in Figure 3 (Camera 1), $635 \mathrm{~mm}$ from the headform. This positioning

11 allowed for a view of a portion of the headform and approximately $140 \mathrm{~mm}$ of the ball

12 trajectory prior to and post impact as per the example extracted still images shown in 13 Figure 4. 


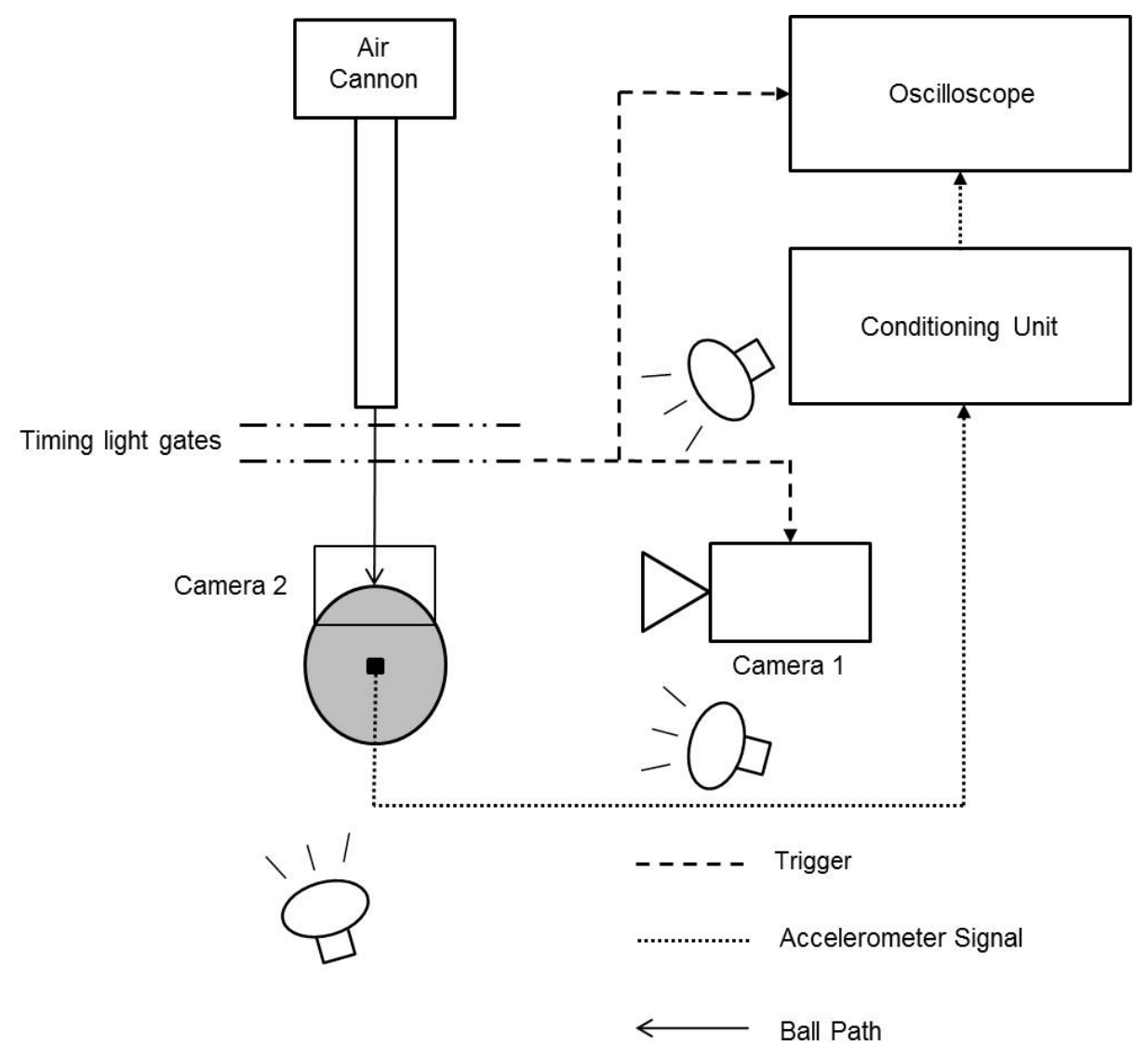

2 Figure 3. Schematic of the experimental arrangement.

3 Prior to each trial, the headform rest position was adjusted, making use of the video

4 camera images to compare the current with previous position, as required to achieve the

5 desired ball-impact location. While this adjustment was always necessary for the

6 Unconstrained, it was rarely required for the Constrained scenario, emphasising why, for

7 ease, this latter mounting arrangement is specified in the Standard tests. Despite careful

8 headform positioning, the ball trajectory out of the cannon was inevitably somewhat

9 variable. A second high-frame-rate camera, a Photron FastCam Ultima APX mono

10 operating at $10 \mathrm{kHz}$, was therefore positioned directly above the impact location, as also

11 shown in Figure 3 (Camera 2), to enable an assessment of the impact location relative to

12 the $y z$-plane, but not used in measurements. By quickly interrogating the two camera 
1 recordings immediately after impact, those for which the ball impact location was

2 substantially different $(\geq 5 \mathrm{~mm})$ to that desired were rejected and a repeated measurement

3 performed. In practice, this impact-position criterion amounted to a repeat of between 1

4 in 5 or 6 impacts which was considered a reasonable compromise between maximising

5 data quality and minimising experiment-completion time. Similarly, variation in ball

6 impact speed with respect to the required nominal was immediately estimated using a

7 pair of timing light gates separated by a distance of $200 \mathrm{~mm}$ with the closest gate

8 positioned $500 \mathrm{~mm}$ from the headform. With respect to this variation, trials were rejected

9 if they deviated by more than $\pm 2 \mathrm{~m} / \mathrm{s}$ from the target impact speed. This deviation led to a

10 similar trial rejection rate to that produced by variations in impact location. It should be

11 noted that actual impact speed values were determined from video data post-processing

12 rather than from the light gates.
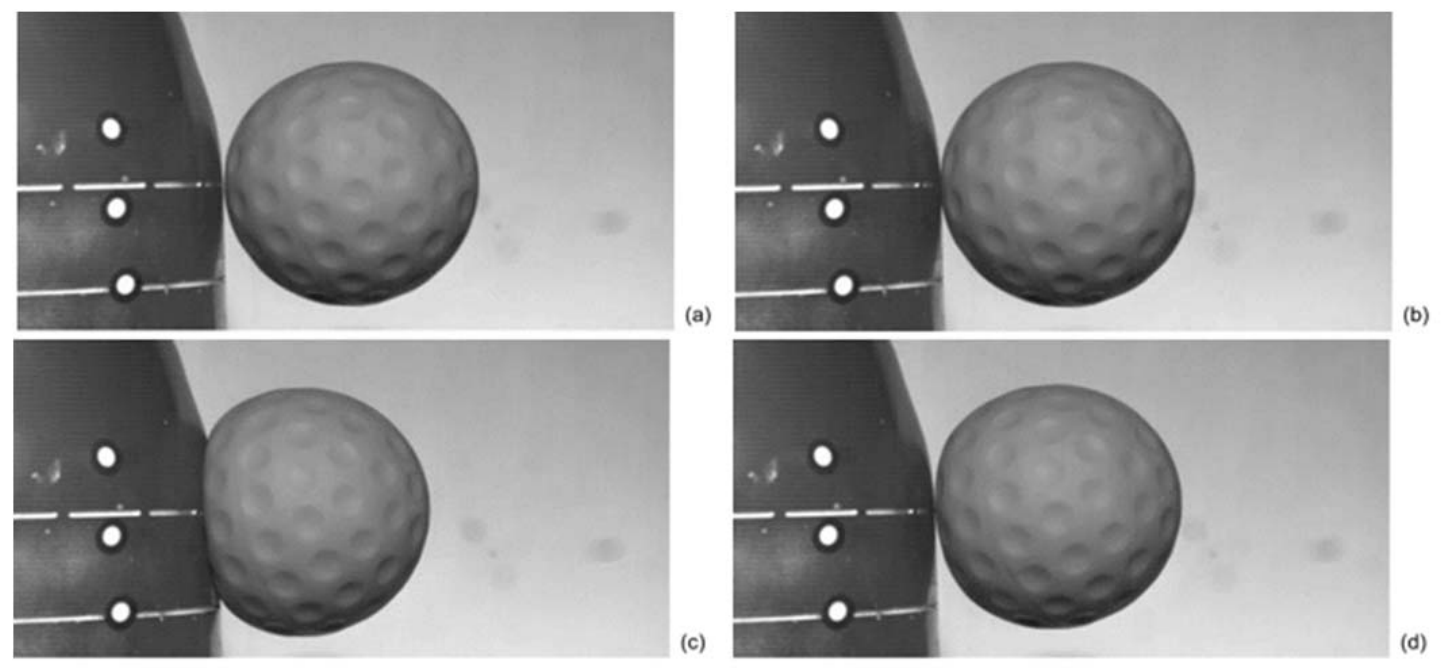

14 Figure 4. Key frames from a typical (Constrained, top, $28 \mathrm{~m} / \mathrm{s}$ ) trial; (a) immediately

15 prior to initial contact, (b) initial contact, (c) maximum ball deformation and (d)

16 immediately following the end of contact. 


\section{Data post-processing}

2

3 A MATLAB script was written and used to enable efficient post-processing of the video

4 data. The script employed edge detection techniques to identify the headform and the ball

5 in each frame. The frame of initial contact (Figure $4 b$ ) was the frame at which the

6 headform edge and ball leading edge first coincided while the frame immediately

7 following the end of contact (Figure 4d) was that in which the two edges separated.

8 Conversion from pixels to mm was performed by using the un-deformed ball diameter -

9 determined using a digital Vernier caliper - at various positions across the image and

10 found to be consistent. Ball deformation was determined on a frame-by-frame basis by

11 determining the straight-line distance between the ball leading and trailing edges in the $z$ -

12 axis (as defined in Figure 1a) with deformation clearly at a maximum when this distance

13 was at a minimum (Figure 4c). By further determining the positions of the three high-

14 contrast circular target markers attached to the headform, as can be seen in Figure 4, the

15 headform positioning, and any variability therein, prior to each impact could be verified.

16 Accelerometer data were processed using Microsoft Excel and MATLAB with the

17 previously set out sensitivities applied therein to convert from V to SI units. Analyses of

18 the data sampled at $50 \mathrm{MHz}$ revealed that no meaningful signal artefacts would be lost by

19 down-sampling by a factor of 100 . Given the accelerometer and video camera data

20 capture were triggered using the same signal from the timing gates, corresponding

21 datasets from each could be and were synchronised with respect to one another. The

22 timestamp of the frames at the beginning and end of ball/headform contact, identified

23 from high-frame-rate video data, were used to enable interrogation of the accelerometer

24 data over that same contact period. The timestamp of the frame of maximum ball 
1 deformation was used to determine the "loading" phase, defined as the period during

2 ball/headform contact leading up to maximum ball deformation, and the "unloading"

3 phase, defined as the contact period immediately following maximum ball deformation

4 until ball/headform separation.

5 Accelerations in the $x-, y$ - and $z$-directions were time-domain integrated using a

6 trapezoidal rule to yield equivalent velocity components; initial non-zero offsets were

7 eliminated by subtracting a mean value for the period of samples one video frame prior to

8 impact so as to de-trend the outputs. Resultant speed traces were interrogated and, using

9 all five trials for a given impact speed and location combination, a mean speed trace was

10 derived (by taking the mean of the five values at each time sample). Interpretation of the

11 video data, as indicated in Figure 4, enabled the frames (and therefore the times) at the

12 start of contact, maximum ball deformation and end of contact, to be determined. The

13 mean speed traces then being possible to be analysed in terms of the loading and

14 unloading phases. Sensitivity analyses were undertaken to determine the impact of

15 selecting a time based on the previous or subsequent frame at all three points of interest

16 with differences in mean speed on the order of $1 \%$ maximum typically observed. During

17 the processing of the accelerometer data, it was found that for one trial (Constrained,

18 Bottom, 25-m/s) accelerometer data had not been recorded correctly and this trial was

19 therefore discarded.

20 Consideration was given to normalisation, for example by deploying a regression 21 analysis, of the results to attempt to account for variation in actual (as opposed to 22 nominal) ball impact speed. Challenges with adopting such an approach, however, 23 include not having a model to be able to confidently predict what the underlying 
1 relationship should be for the regression analysis. Furthermore, normalisation to a

2 singular value of impact speed would have clearly rendered tests for statistical

3 significance of results impossible.

4 Where statistical significance tests have been completed, these were conducted using

5 IBM SPSS version 22. Given that only two scenarios, Constrained and Unconstrained,

6 are under consideration, multiple comparison tests are not relevant. Accordingly,

7 following confirmation of normal distribution and homogeneity of variance using

8 Shapiro-Wilk and Levene's tests, respectively, independent t-tests were used to identify

9 differences between the two scenarios for the various impact conditions. Initially, the

10 alpha level was set to 0.05 . However, to adjust for family-wise errors that may occur due

11 to the 45 independent t-tests, a Holm-Bonferroni correction was subsequently applied

12 reducing the alpha level to 0.0011 .

\section{Results}

\section{Contact duration}

15

16 The contact durations observed from high-frame-rate video recordings show no clear

17 separation between equivalent speed/location impacts for Constrained ("Cons.-xxx") vs.

18 Unconstrained ("Uncon.-xxx") scenarios as can be seen in Figure 5 and Table 1. It is

19 evident from independent t-tests conducted that the only statistically significant

20 difference between the Constrained and Unconstrained conditions occurs at the Middle-

$2125-\mathrm{m} / \mathrm{s}$ condition $(\mathrm{P}=0.0001)$. While, upon initial inspection of these results, it may

22 appear that increasing inbound ball speed leads to slightly shorter contact durations,

23 standard deviations are significant relative to the differences between the means, as can

24 be observed in Table 1. From the distribution of the data points in Figure 5, it is also 
1 evident that variation in contact duration is generally reduced as the inbound speed

2 increases, with the least variation at $28-\mathrm{m} / \mathrm{s}$ nominal ball speed. It is further evident from

3 the standard deviations that this reduction in contact time is the case for the majority of

4 impact location/mounting arrangement combinations, with the exception of Constrained-

5 Top and Unconstrained-Bottom.

6 The largest variation within a specific testing condition was for Unconstrained-Top, 22-

$7 \mathrm{~m} / \mathrm{s}$ nominal ball speed. Limited variation in contact duration may be the result of the

8 limited resolution to identify the key frames where contact begins and ends. Contact

9 duration variation determination is also a function of variation in the impact location

10 laterally which, due to the curved nature of the headform, may lead to small but

11 measureable differences in determined contact duration. At the bottom impact location,

12 however, the headform surface profile is much flatter and deviations laterally should not

13 result in as significant a change in the determined contact duration than may be the case

14 at the top and middle locations. The largest difference between the Constrained and

15 Unconstrained scenarios occurred at the Middle, $25-\mathrm{m} / \mathrm{s}$ nominal ball speed, where the

16 Constrained scenario displayed a mean contact duration $0.14 \mathrm{~ms}$ greater than that of the

17 Unconstrained equivalent. 


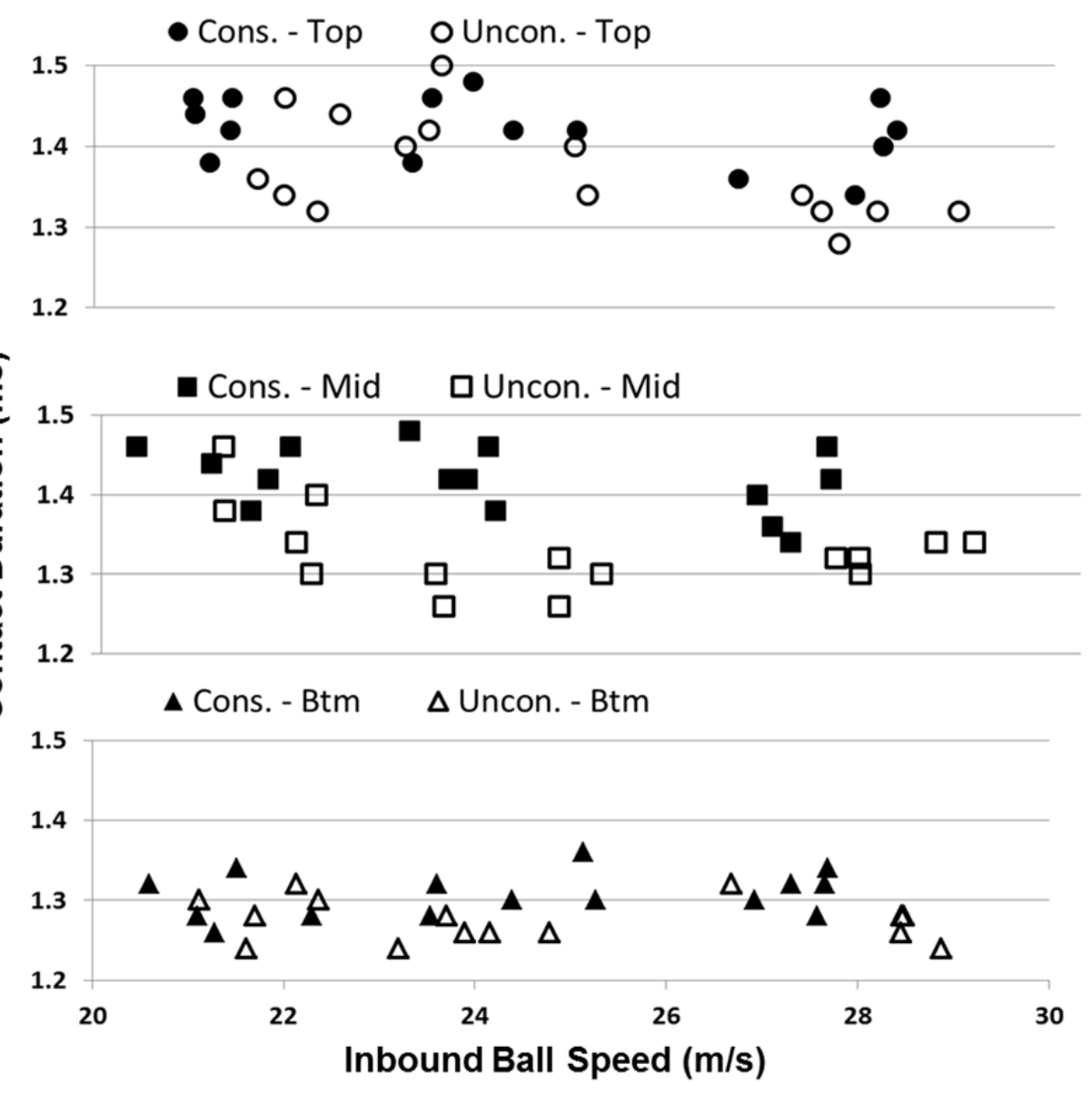

2 Figure 5. Comparison between contact durations and measured inbound ball speeds.

3 Table 1. Means and standard deviations for contact durations and measured inbound ball 4 speeds.

\begin{tabular}{ccccccccc}
\hline & \multicolumn{3}{c}{ Constrained } & \multicolumn{3}{c}{ Unconstrained } \\
\hline \multirow{2}{\text{Impact}}{$\begin{array}{c}\text { Ball Speed } \\
(\mathbf{m} / \mathbf{s})\end{array}$} & $\begin{array}{c}\text { Contact Duration } \\
(\mathbf{m s})\end{array}$ & $\begin{array}{c}\text { Ball Speed } \\
(\mathbf{m} / \mathbf{s})\end{array}$ & $\begin{array}{c}\text { Contact Duration } \\
(\mathbf{m s})\end{array}$ \\
Location & Mean & SD & Mean & SD & Mean & SD & Mean & SD \\
\hline \multirow{2}{*}{ Top } & 27.9 & 0.6 & 1.40 & 0.04 & 27.6 & 0.6 & 1.32 & 0.02 \\
& 24.2 & 0.5 & 1.43 & 0.03 & 24.0 & 0.8 & 1.41 & 0.05 \\
& 21.3 & 0.2 & 1.43 & 0.03 & 22.0 & 0.4 & 1.38 & 0.06 \\
\hline \multirow{3}{*}{ Middle } & 27.1 & 0.3 & 1.40 & 0.04 & 28.1 & 0.8 & 1.32 & 0.01 \\
& 23.6 & 0.4 & 1.43 & 0.03 & 24.0 & 0.8 & 1.29 & 0.02 \\
& 21.3 & 0.6 & 1.43 & 0.03 & 21.8 & 0.4 & 1.38 & 0.05 \\
\hline \multirow{3}{*}{ Bottom } & 27.4 & 0.3 & 1.31 & 0.02 & 28.1 & 0.6 & 1.28 & 0.03 \\
& 24.2 & 0.6 & 1.31 & 0.03 & 24.0 & 0.7 & 1.26 & 0.01 \\
& 21.5 & 0.6 & 1.30 & 0.03 & 21.6 & 0.5 & 1.29 & 0.03 \\
\hline
\end{tabular}




\section{Ball deformation}

2

3 When comparing the duration from initial contact to maximum ball deformation, as

4 shown in Table 2, it appears that this contact time is slightly greater for the Constrained

5 scenario than for the Unconstrained. However, when the standard deviations are also

6 considered, it is apparent that, generally, there is little difference between equivalent

7 scenarios for the two mounting arrangements. This lack of any statistically significant

8 difference is confirmed by independent t-tests between the two scenarios for all impact

9 conditions.

10 Table 2. Means and standard deviations for times from initial contact to maximum ball

11 deformation (inbound ball speed equivalents included again for completeness).

\begin{tabular}{|c|c|c|c|c|c|c|c|c|}
\hline & \multicolumn{4}{|c|}{ Constrained } & \multicolumn{4}{|c|}{ Unconstrained } \\
\hline & \multicolumn{2}{|c|}{$\begin{array}{l}\text { Ball Speed } \\
(\mathrm{m} / \mathrm{s})\end{array}$} & \multicolumn{2}{|c|}{$\begin{array}{l}\text { Time to Max } \\
\text { Ball Def. }(\mathrm{ms})\end{array}$} & \multicolumn{2}{|c|}{$\begin{array}{l}\text { Ball Speed } \\
(\mathrm{m} / \mathrm{s})\end{array}$} & \multicolumn{2}{|c|}{$\begin{array}{l}\text { Time to Max } \\
\text { Ball Def. (ms) }\end{array}$} \\
\hline & Mean & SD & Mean & SD & Mean & SD & Mean & SD \\
\hline \multirow{3}{*}{ Top } & 27.9 & 0.6 & 0.57 & 0.03 & 27.6 & 0.6 & 0.55 & 0.02 \\
\hline & 24.2 & 0.5 & 0.62 & 0.04 & 24.0 & 0.8 & 0.55 & 0.03 \\
\hline & 21.3 & 0.2 & 0.62 & 0.03 & 22.0 & 0.4 & 0.55 & 0.04 \\
\hline \multirow{3}{*}{ Middle } & 27.1 & 0.3 & 0.57 & 0.02 & 28.1 & 0.8 & 0.55 & 0.02 \\
\hline & 23.6 & 0.4 & 0.59 & 0.04 & 24.0 & 0.8 & 0.54 & 0.02 \\
\hline & 21.3 & 0.6 & 0.54 & 0.06 & 21.8 & 0.4 & 0.54 & 0.03 \\
\hline \multirow{3}{*}{ Bottom } & 27.4 & 0.3 & 0.56 & 0.07 & 28.1 & 0.6 & 0.50 & 0.01 \\
\hline & 24.2 & 0.6 & 0.61 & 0.04 & 24.0 & 0.7 & 0.53 & 0.03 \\
\hline & 21.5 & 0.6 & 0.56 & 0.07 & 21.6 & 0.5 & 0.53 & 0.04 \\
\hline
\end{tabular}

12

13 As should be expected and as can be clearly observed in Figure 6, for both the

14 Constrained and Unconstrained mounting arrangement scenarios, the maximum ball

15 deformation increased with increasing inbound ball speed. From the distribution of these

16 data, it would appear that, despite relatively large variations possibly due to the limited

17 spatial pixel resolution of the video data from which they are derived, generally greater

18 ball deformation occurs in the Constrained scenarios compared to their Unconstrained

19 equivalents. Table 3 shows the means and standard deviations for minimum ball 
1 diameters, i.e. at maximum ball deformation, against mean ball speeds and, with the

2 exception of $28-\mathrm{m} / \mathrm{s}$ impacts at the Middle impact location, the Constrained scenario

3 displayed slightly greater mean ball deformation at all combinations of impact location

4 and nominal speed. Despite the differences between the Constrained and the

5 Unconstrained trials presented in Figure 6, Table 3 confirms that these differences are

6 generally relatively small with relatively high standard deviations. Independent t-tests

7 identified the differences in ball deformations between the Constrained and

8 Unconstrained scenarios to be only statistically significant at the Bottom, $28-\mathrm{m} / \mathrm{s}$

9 combination $(\mathrm{P}=0.001)$.

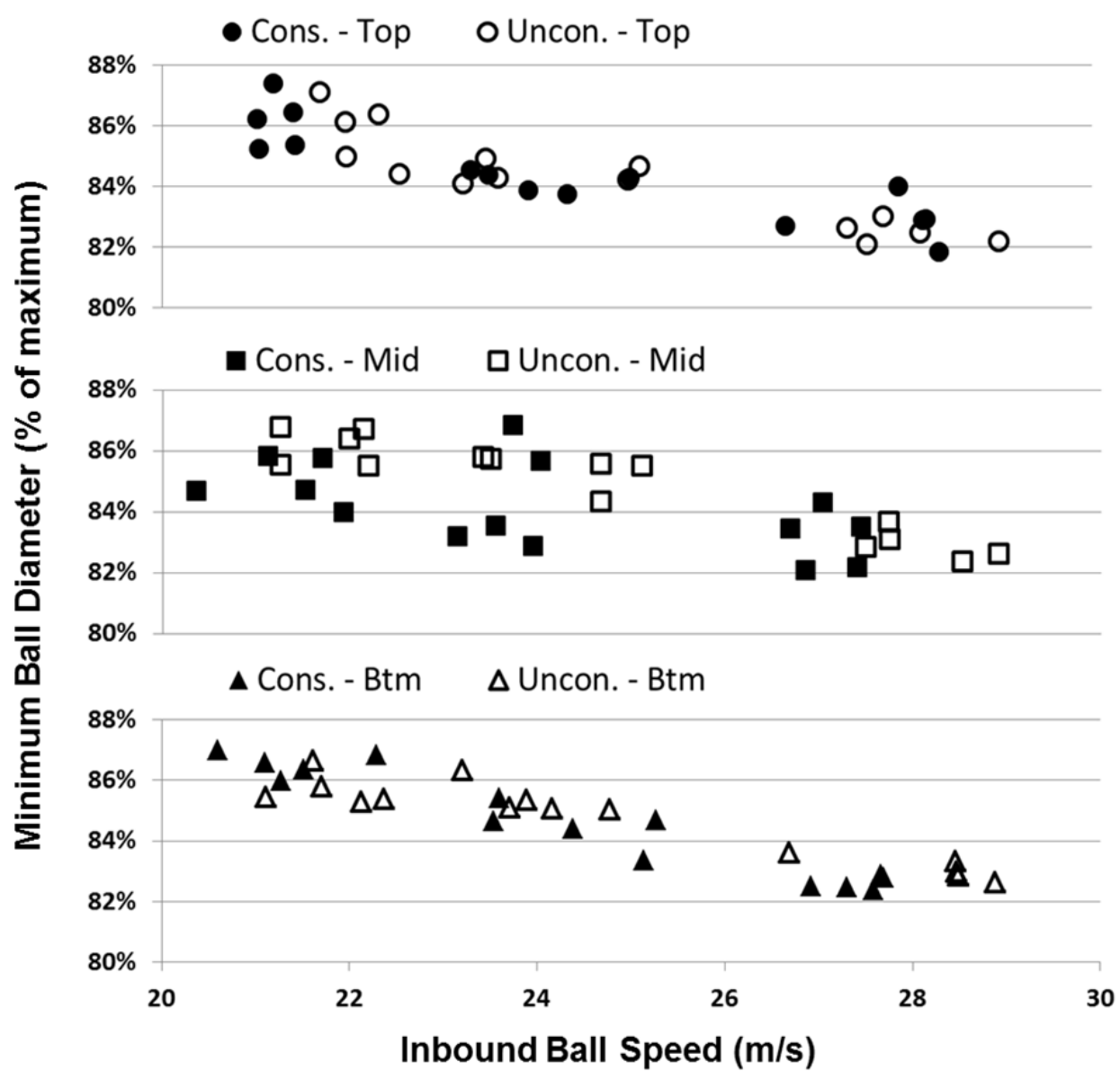

10

11 Figure 6. Comparison between minimum ball diameters and measured inbound ball 12 speeds. 
1 Table 3. Means and standard deviations for minimum ball diameters while in contact with

2 the headform (inbound ball speed equivalents included again for completeness).

\begin{tabular}{|c|c|c|c|c|c|c|c|c|}
\hline \multirow{3}{*}{$\begin{array}{l}\text { Impact } \\
\text { Location }\end{array}$} & \multicolumn{4}{|c|}{ Constrained } & \multicolumn{4}{|c|}{ Unconstrained } \\
\hline & \multicolumn{2}{|c|}{$\begin{array}{c}\text { Impact Speed } \\
(\mathrm{m} / \mathrm{s})\end{array}$} & \multicolumn{2}{|c|}{$\begin{array}{l}\text { Min Ball Diameter } \\
\text { (Relative to Max) }\end{array}$} & \multicolumn{2}{|c|}{$\begin{array}{c}\text { Impact Speed } \\
(\mathrm{m} / \mathrm{s})\end{array}$} & \multicolumn{2}{|c|}{$\begin{array}{l}\text { Min Ball Diameter } \\
\text { (Relative to Max) }\end{array}$} \\
\hline & Mean & SD & Mean & SD & Mean & SD & Mean & SD \\
\hline \multirow{3}{*}{ Top } & 27.9 & 0.6 & $82.8 \%$ & $0.6 \%$ & 27.6 & 0.6 & $83.6 \%$ & $0.4 \%$ \\
\hline & 24.2 & 0.5 & $83.9 \%$ & $0.4 \%$ & 24.0 & 0.8 & $84.7 \%$ & $0.6 \%$ \\
\hline & 21.3 & 0.2 & $85.9 \%$ & $0.7 \%$ & 22.0 & 0.4 & $86.1 \%$ & $1.3 \%$ \\
\hline \multirow{3}{*}{ Middle } & 27.1 & 0.3 & $83.2 \%$ & $1.0 \%$ & 28.1 & 0.8 & $82.8 \%$ & $0.9 \%$ \\
\hline & 23.6 & 0.4 & $84.8 \%$ & $1.1 \%$ & 24.0 & 0.8 & $86.2 \%$ & $0.9 \%$ \\
\hline & 21.3 & 0.6 & $85.1 \%$ & $0.8 \%$ & 21.8 & 0.4 & $86.1 \%$ & $0.9 \%$ \\
\hline \multirow{3}{*}{ Bottom } & 27.4 & 0.3 & $82.5 \%$ & $0.3 \%$ & 28.1 & 0.6 & $83.5 \%$ & $0.3 \%$ \\
\hline & 24.2 & 0.6 & $84.7 \%$ & $0.5 \%$ & 24.0 & 0.7 & $85.3 \%$ & $1.0 \%$ \\
\hline & 21.5 & 0.6 & $86.2 \%$ & $0.9 \%$ & 21.6 & 0.5 & $86.4 \%$ & $0.5 \%$ \\
\hline
\end{tabular}

4 Headform acceleration

6 In all equivalent mounting arrangement scenarios, mean resultant acceleration during the

7 loading phase was found to increase with inbound ball speed, as can be readily observed

8 in Figure 7. The Constrained and Unconstrained groups of data points are distributed

9 similarly and over a similar range, particularly for the $28-\mathrm{m} / \mathrm{s}$ ball speed impacts. Such

10 characteristics suggest similar measurement variabilities and uncertainties for each

11 arrangement and that, therefore, differences between the derived measures are not a

12 function of different aspects of the two sets of experiments, for example substantial

13 differences in the projection of the ball for the Constrained and Unconstrained setups. At

$1422-\mathrm{m} / \mathrm{s}$, there appears to be greater separation between the Constrained and

15 Unconstrained data points, indicating that the headform displayed slightly greater mean

16 accelerations in the Unconstrained scenarios. These observations are reflected in the

17 mean average resultant acceleration statistics included in Table 4, where there are

18 generally no distinct differences between the Constrained and Unconstrained equivalent 
1 scenarios particularly at $28-$ and $25-\mathrm{m} / \mathrm{s}$ ball speed. There are, however, differences at 22-

$2 \mathrm{~m} / \mathrm{s}$ as the Unconstrained data exhibit a higher mean resultant acceleration at both the

3 Top and Middle impact locations. Figure 7 suggests that, where variability was high,

4 these differences in mean resultant acceleration are due to genuine distribution of data

5 points, as opposed to outliers skewing the statistics. The only statistically significant

6 difference between the scenarios was found at an impact speed of $22-\mathrm{m} / \mathrm{s}$ at the Top

7 impact location $(\mathrm{P}<0.001)$.

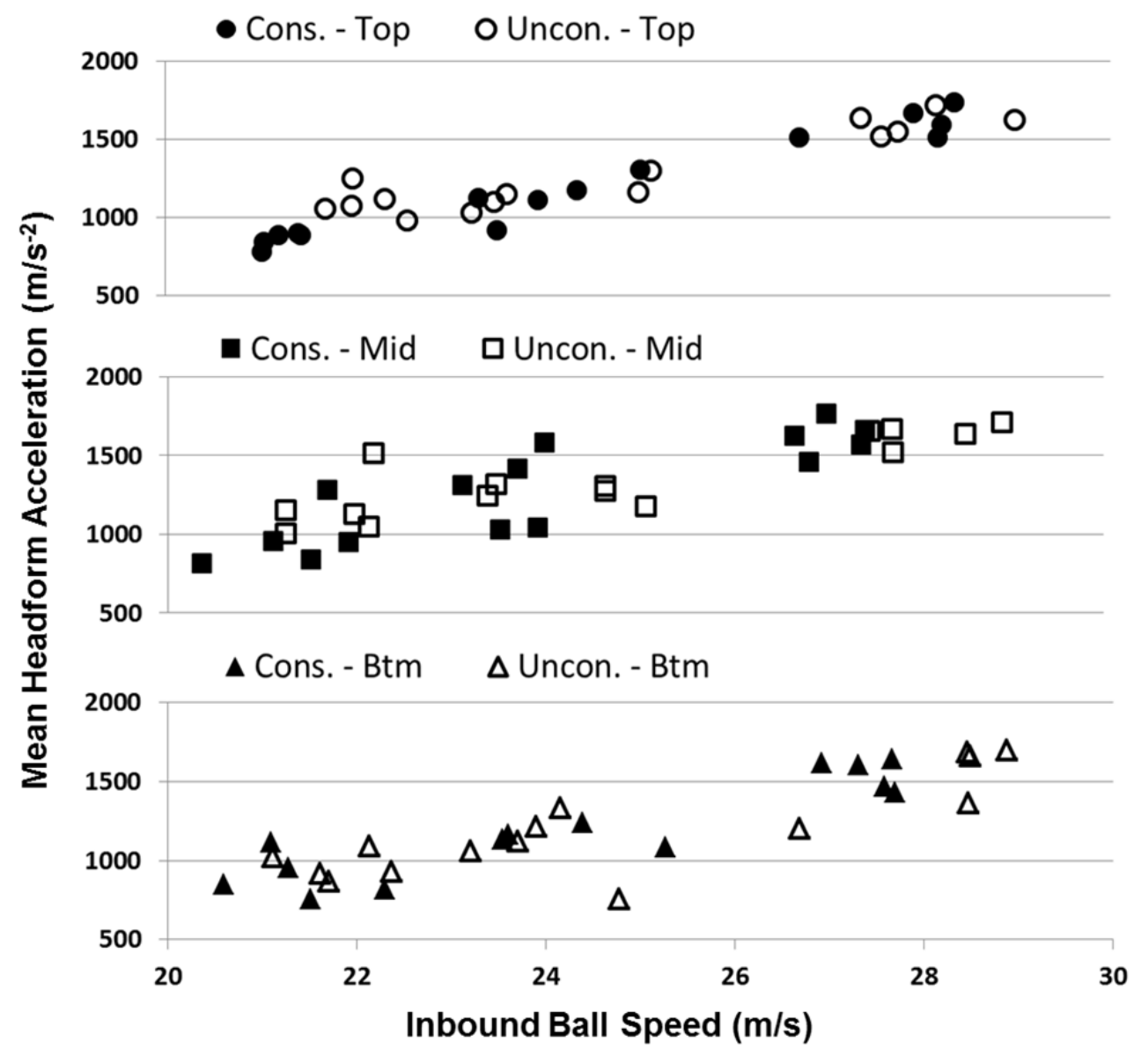

9 Figure 7. Comparison between mean resultant accelerations during the loading phase and 10 measured inbound ball speeds.

11

12 
1 Table 4. Means and standard deviations of mean resultant accelerations during the

2 loading phase (inbound ball speed equivalents included again for completeness).

\begin{tabular}{|c|c|c|c|c|c|c|c|c|}
\hline \multirow[b]{3}{*}{$\begin{array}{l}\text { Impact } \\
\text { Location }\end{array}$} & \multicolumn{4}{|c|}{ Constrained } & \multicolumn{4}{|c|}{ Unconstrained } \\
\hline & \multicolumn{2}{|c|}{$\begin{array}{c}\text { Impact } \\
\text { Speed }(\mathrm{m} / \mathrm{s})\end{array}$} & \multicolumn{2}{|c|}{$\begin{array}{c}\text { Mean Resultant } \\
\text { Acceleration }\left(\mathrm{m} / \mathrm{s}^{2}\right)\end{array}$} & \multicolumn{2}{|c|}{$\begin{array}{c}\text { Impact } \\
\text { Speed }(\mathrm{m} / \mathrm{s})\end{array}$} & \multicolumn{2}{|c|}{$\begin{array}{c}\text { Mean Resultant } \\
\text { Acceleration }\left(\mathrm{m} / \mathrm{s}^{2}\right)\end{array}$} \\
\hline & Mean & SD & Mean & SD & Mean & SD & Mean & SD \\
\hline \multirow{4}{*}{ Top } & 27.9 & 0.6 & 1606 & 88 & 27.6 & 0.6 & 1610 & 72 \\
\hline & 24.2 & 0.5 & 1127 & 126 & 24.0 & 0.8 & 1149 & 87 \\
\hline & 21.3 & 0.2 & 860 & 45 & 22.0 & 0.4 & 1096 & 88 \\
\hline & 27.1 & 0.3 & 1615 & 101 & 28.1 & 0.8 & 1638 & 63 \\
\hline
\end{tabular}

Middle 